\title{
El principio de participación económica de los miembros de la cooperativa desde una perspectiva histórica y doctrinal ${ }^{1}$
}

\author{
Alejandro Martínez Charterina \\ Catedrático Emérito de la Universidad de Deusto
}

Sumario: 1. El tercer principio cooperativo y sus antecedentes. 2. Análisis del principio de participación económica de los socios. 3. Relación del principio con los valores cooperativos. 4. Inquietudes y planteamientos recientes relativos a las cooperativas y el capital. 5. Bibliografía.

Resumen: El principio de participación económica de los miembros de la cooperativa recoge los principios contemplados desde el origen del cooperativismo moderno de interés limitado al capital y reparto de los excedentes en función de la actividad cooperativizada realizada por los socios. Pero además afirma la contribución equitativa al capital de la cooperativa por parte de los socios y su gestión democrática, y deja abierta la necesidad de un capital fuerte y estable que pueda permitir el funcionamiento de la empresa a lo largo del tiempo, así como los problemas que son susceptibles de acompañarle.

Palabras clave: cooperativas, identidad cooperativa, principios cooperativos.

Abstract: The principle of economic participation of cooperative members adheres to the principles underpinning the origins of modern cooperativism, based on limited interest on capital and the distribution of surplus among members according to the amount of cooperative activities performed. It also strengthens the equitable contribution to cooperatives' capital by members and their democratic management, and leaves open the need for a strong and stable capital base that allows for the good running of the business over time and its related problems.

Keywords: Cooperatives, cooperative identity, cooperative principles.

1 Texto basado en la ponencia del mismo título presentada en el Congreso de la Asociación Internacional de Derecho Cooperativo y la Academia Vasca de Derecho sobre «El principio de participación económica de los miembros. Consecuencias jurídicas y económicas derivadas de su aplicación práctica», en Bilbao, el 29 de noviembre de 2017. 


\section{El tercer principio cooperativo y sus antecedentes}

El tercero de los principios cooperativos de la Declaración de la Alianza Cooperativa Internacional sobre la Identidad Cooperativa se titula "participación económica de los socios» y presenta una redacción larga y compleja. El texto es el siguiente:

«Los socios contribuyen equitativamente al capital de sus cooperativas y lo gestionan de forma democrática. Por lo menos una parte de ese capital es normalmente propiedad común de la cooperativa. Usualmente, los socios reciben una compensación, si la hay, limitada sobre el capital entregado como condición para ser socio. Los socios asignan los excedentes a todos o alguno de los siguientes fines: el desarrollo de su cooperativa, posiblemente mediante el establecimiento de reservas, parte de las cuales por lo menos serían irrepartibles; el beneficio de los socios en proporción a sus operaciones con la cooperativa; y el apoyo de otras actividades aprobadas por los socios. $\rangle^{2}$

Este principio agrupa dos de los principios cooperativos originales, procedentes de la cooperativa de Rochdale, que Paul Lambert recoge como «... 3) pago de un interés limitado al capital; 4) retorno de los excedentes a los miembros en proporción a sus compras; ;... $\gg^{3}$. El artículo 22 de los Estatutos de Rochdale fija el interés al capital en el $3,5 \%$ anual, y el reparto de los beneficios restantes tras el pago del interés entre los miembros en proporción a los gastos realizados en el almacén ${ }^{4}$. El interés del 3,5\% sería elevado al 5\% anual en las enmiendas a los Estatutos de $1845^{5}$.

En la primera reforma de los principios cooperativos, que tuvo lugar en el XV Congreso de la Alianza Cooperativa Internacional, celebrado en París en 1937, en la que se consideraron dos categorías de principios, cuatro necesarios para que una cooperativa pudiera ser con-

2 I.C.A.: Declaración de la Alianza Cooperativa Internacional sobre la Identidad Cooperativa. Los principios cooperativos, Consejo Superior de Cooperativas de Euskadi, Vitoria-Gasteiz, 1996, p. 19.

3 Lambert, Paul: La doctrina cooperativa, 3. ${ }^{a}$ ed., Intercoop, Buenos Aires, 1970, pp. 57.

4 Laws and objects of the Rochdale Society of Equitable Pionners: enrolled according to the acts, $10^{\text {th }}$, George $N$, and $4^{\text {th }}$ and $5^{\text {th }}$, William IV. Printed by Jesse Hall, Rochdale, 1844, p. 9.

5 Supplement of Amended Laws of the First Department of the Rochdale Society of Equitable Pioneers, held in Toad-Lane, in the Parish of Rochdale, in the County of Lancaste. Printed by Jesse Hall, Rochdale, 1845, p. 5. 
siderada como tal, y aceptada su afiliación a la Alianza a través de los organismos federativos correspondientes, y tres simplemente recomendables, la distribución de los excedentes a prorrata de las transacciones realizadas y el interés limitado al capital formaron parte de los cuatro necesarios junto con la libre adhesión y el control democrático.

Del mismo modo, en la segunda reforma, que se produjo en el XXIII Congreso de la Alianza, celebrado en Viena en 1966, constitutiva justamente de la formulación inmediatamente anterior a la actual, el interés limitado al capital y el reparto de los excedentes en proporción a las transacciones efectuadas eran los principios tercero y cuarto.

También en Viena se eliminó el principio originario de Rochdale de las compras y ventas al contado, que ya había quedado relegado a simplemente recomendable en la reforma de $1937^{6}$.

En el Congreso previo y preparatorio del Centenario de la Alianza que aprobó la Declaración sobre la identidad cooperativa, que fue el XXX Congreso celebrado en Tokio en octubre de 1992, el Informe Böök recomendaba que se fusionaran los dos principios señalados en uno sólo sobre la formación del capital de la cooperativa7.

Y atendiendo a la recomendación de Sven Ake Böök quedó aprobado el principio agrupando los dos anteriores.

\section{Análisis del principio de participación económica de los socios}

Además de la fusión de los principios de interés limitado al capital y de reparto de los excedentes en función de la actividad efectuada, el principio comienza por hacer una declaración sobre el capital.

La primera frase del texto señala la necesidad de que los socios realicen de forma equitativa sus aportaciones para la formación del capital de la cooperativa, capital que, recuerda el texto, se deberá gestionar democráticamente en sintonía con el segundo de los principios, el de gestión democrática.

E inmediatamente señala que normalmente parte del capital es propiedad común de la cooperativa, lo que viene a constituir una declaración novedosa sobre el capital de la empresa cooperativa.

6 A.C.I.: Notas de orientación para los principios cooperativos. Alianza Cooperativa Internacional, 2015, p. 31.

7 Böök, Sven Ake: Co-operative values in a changing world (report to the ICA Congress, Tokio, October 1992), International Co-operative Alliance, Geneva, 1992, p. 17. 
Esta noción de propiedad colectiva del capital, especialmente importante para las cooperativas de trabajadores, se introdujo a través de una enmienda de la delegación francesa ${ }^{8}$.

La cooperativa, como empresa que es, precisa capital para su funcionamiento. Y lo razonable es que los socios aporten ese capital, toda vez que la cooperativa ha de gestionarse democráticamente por ellos, lo que dificulta la obtención de capital externo a la cooperativa y, en principio, ajeno a la gestión de ese capital.

El Informe que acompaña a la Declaración ${ }^{9}$ presenta cuatro formas de aportación de capital de los socios a la cooperativa:

- Al incorporarse como socio a la misma cada miembro hace una aportación al capital.

- A través de la dotación de reservas. Cuando las cooperativas obtienen beneficios pueden destinar parte de ellos a constituir y acrecentar las reservas, ya sea en cumplimiento de la legislación aplicable cuando lo exige, o por decisión voluntaria. Cabe interpretar la dotación de estas reservas como la representación del logro colectivo de los socios y, en ocasiones en consecuencia, no generan intereses ni son distribuibles entre los socios.

- Mediante la capitalización de retornos cooperativos. De este modo los beneficios a repartir entre los socios quedan en todo o parte en la cooperativa como capital de la misma. Estas aportaciones no generan intereses sino que fortalecen a la cooperativa empresa.

- Por fin realizando aportaciones especiales al capital de la sociedad cuando la cooperativa lo solicita. Generalmente estas aportaciones si generan intereses no especulativos.

Inmediatamente después de anunciar la contribución equitativa de los socios al capital de la cooperativa el texto informa de que normalmente una parte del capital es propiedad común de la cooperativa.

Esta forma de redacción, «por lo menos parte de ese capital es normalmente propiedad común de la cooperativa» ${ }^{10}$, pone de manifiesto la idea de la necesidad del capital y de que sea permanente, juntamente con la noción de propiedad colectiva del capital.

El principio de adhesión voluntaria y abierta hace de la cooperativa una sociedad de capital variable, mayor o menor en función de la entrada y salida de socios.

8 A.C.I.: Notas de orientación..., o.c., p. 31.

9 I.C.A.: Declaración..., o.c., pp. 55-57.

10 El subrayado es mío para llamar la atención de la redacción concreta. 
Por otro lado en la parte en que el capital provenga de los beneficios destinados al mismo, es decir, de la retribución de la actividad empresarial, que es justamente la gestión democrática colectivamente realizada en la cooperativa, es el conjunto de los socios, no cada socio individualmente considerado, el que ha realizado esa aportación.

Este hecho justifica que las disposiciones legislativas puedan considerar que en el supuesto de liquidación ese capital colectivo no se pueda repartir entre los socios, pudiendo destinarse, en cambio, a la promoción y fomento del cooperativismo, o al fortalecimiento de otras cooperativas.

Desde otra consideración complementaria, el capital de la cooperativa es también un nexo de unión del tiempo en el que van sustituyéndose unos socios por otros, y constituye un elemento importante de estabilidad y permanencia.

Tras esta introducción sobre la aportación de capital por los socios y la importancia del mismo y de su estabilidad, el texto del principio aborda su retribución a través de un interés limitado. Los socios reciben una compensación limitada sobre el capital entregado para ser socio «usualmente» y «si la hay».

Por consiguiente cabe retribuir al capital con un interés limitado o no retribuirlo. De las formas en que el socio aporta capital, que hemos visto, no se retribuirá el procedente de la dotación de reservas y la capitalización de retornos. Cabe hacerlo para el capital entregado como condición para ser socio, así como para las aportaciones especiales, pero con un interés limitado, que en los términos del Informe que acompaña a la Declaración de la Alianza ha de ser «... un tipo justo. La renta pagada... debe ser de un tipo competitivo y no especulativo: por ejemplo, el tipo de interés gubernamental o bancario normal»» ${ }^{11}$.

Cabe considerar que los socios no aportan capital a su cooperativa para obtener una ganancia con esa aportación. Lo hacen porque la cooperativa, al igual que cualquier otra empresa, necesita capital para poder llevar a cabo su actividad. Y de ello se desprende que ese capital pueda no ser retribuido, y que cuando exista una retribución sea limitada. "Se juntan el capital y la actividad empresarial, pero no se confunde el interés que retribuye el capital, que es fijo, y en la cooperativa además limitado, con el beneficio variable que corresponde al empresario. No se reparte en la cooperativa, a diferencia de lo que sucede en la sociedad capitalista, el dividendo, suma de interés y beneficio» ${ }^{12}$.

11 I.C.A.: Declaración..., o.c., p. 57.

12 Martínez Charterina, Alejandro: La cooperativa y su identidad, Dykinson, Madrid, 2016, p. 64. 
A diferencia de lo que sucede en la empresa capitalista en la que se unen los dos factores de producción, el capital y la actividad empresarial, en la misma persona que recibe como retribución el dividendo, suma de interés del capital y beneficio empresarial, la cooperativa separa el interés del capital y el beneficio empresarial aunque sean las mismas personas las que gestionan la empresa y aportan el capital.

El capital y la actividad empresarial reciben una retribución en cuanto que, como factores de producción, participan en el proceso productivo. El capital recibe el interés y el empresario el beneficio. El interés, al igual que la renta de la tierra y el salario del trabajo, es una retribución fija, que paga el empresario que organiza la actividad productiva. El beneficio que recibe el empresario como retribución es, en cambio, residual y variable ${ }^{13}$.

En la empresa capitalista se junta el interés fijo y el beneficio variable, haciendo que la suma, el dividendo, sea variable. En la cooperativa se separa el interés del capital y el beneficio empresarial aunque confluyan en las mismas personas. Así el interés es fijo y además limitado, como señala el principio, mientras que el beneficio es variable.

Finalmente el texto del principio se ocupa del reparto del beneficio, que en la terminología habitual que se usa en el cooperativismo se denomina excedentes, es decir lo que queda.

El texto nos indica que los excedentes pueden ser destinados por parte de los socios a algunas o a todas las finalidades siguientes: al desarrollo de la cooperativa, al reparto entre los socios, y a la realización de las actividades que consideren oportunas.

Son los socios, por consiguiente, los que determinan el destino de los excedentes.

En primer lugar, pueden dotar fondos de reserva para favorecer el desarrollo de la cooperativa y fortalecerla; e insiste en que esas reservas sean irrepartibles en todo o, al menos, en parte, bajo la consideración, señalada anteriormente, de que se trata de un logro colectivo de los socios.

En segundo lugar pueden repartirse entre los socios en forma de retorno. En la medida en la que han contribuido a la obtención del beneficio, reciben la parte correspondiente del mismo.

En este sentido, el reparto del retorno entre los socios será proporcional a la participación del socio en la cooperativa, es decir, al consumo realizado si se trata de una cooperativa de consumo, al trabajo

13 Puede verse sobre los factores de producción y su retribución MARTínez CHARTERINA, Alejandro: Introducción a la Economía, 2. ${ }^{a}$ edición, Dykinson, Madrid, 2017, pp. 26-28. 
realizado si es una cooperativa de trabajo, y, con carácter general, a la aportación de cada socio al funcionamiento de la cooperativa del género que esta sea.

Pero en ningún caso en proporción al capital aportado que, como venimos considerando, queda al margen de la consecución y reparto del beneficio en la cooperativa.

Por fin, los socios pueden determinar que el beneficio se destine, en todo o en parte, al apoyo de cualquier otra actividad. El Informe que acompaña a la Declaración sugiere que una de estas actividades puede ser desarrollo del movimiento cooperativo en todos sus niveles, «local, nacional, regional e internacional» ${ }^{14}$.

En este sentido, es también habitual que las cooperativas fomenten actividades de carácter formativo para sus socios, sus familias y su entorno.

\section{Relación del principio con los valores cooperativos}

La idea de que los principios cooperativos deben considerarse en su conjunto, como una unidad que resulta ser la propia cooperativa, de forma que no hay unos principios más importantes que otros individualmente considerados, puede ser también aplicable a los valores que, en cuanto aspiraciones a las que tiende la cooperativa y que pretende alcanzar justamente a través de los principios, también pueden considerarse como un todo.

Al mismo tiempo cabe pensar que, considerando los valores como expresiones valiosas de las conductas, unos valores conducen a los otros a medida que se van alcanzando con la práctica.

Dicho esto, sin embargo, quisiera proponer una reflexión de conexión de este tercer principio del capital especialmente con algunos de los valores operativos como la democracia, equidad y solidaridad, así como con los valores éticos de responsabilidad y vocación sociales.

La misma redacción del principio comienza por destacar la gestión democrática del capital aportado por los socios.

«Los socios contribuyen equitativamente al capital de sus cooperativas...», comienza el tercer principio. Además el trato equitativo de los socios se manifiesta en la forma de recompensar su participación en el reparto del beneficio ${ }^{15}$.

\footnotetext{
14 I.C.A.: Declaración..., o.c., p. 59.

15 Puede verse en este sentido el Informe que acompaña a la Declaración de la Alianza, en I.C.A.: Declaración..., o.c., p. 39.
} 
Del mismo modo la solidaridad, muy vinculada al valor de la autoayuda, entendida como ayuda mutua, se materializa en el principio al entender que la cooperativa, como señala en Informe de la Declaración, "es más que una asociación de socios; es también una colectividad» ${ }^{16}$. Así se comprende el interés de los socios por mantener sus aportaciones al capital a lo largo del tiempo, a dotar reservas irrepartibles, y a apoyar el desarrollo de un movimiento cooperativo, en suma a mirar hacia el futuro y trascender el presente en el fortalecimiento de la cooperativa.

En este mismo sentido se comprende la especial vinculación de este tercer principio de la participación económica de los socios a los valores éticos de responsabilidad y vocación sociales.

Las cooperativas se forman con personas de una comunidad que tratan de ayudarse a través de la empresa que forman, y asumen el cometido de actuar mirando a la sociedad en la que desempeñan su actividad de forma responsable. Del mismo modo en muchas ocasiones la vocación social les lleva a emplear sus beneficios en favor de la comunidad más próxima, o incluso más alejada mediante sus colaboraciones con el desarrollo cooperativo en los países más desfavorecidos ${ }^{17}$.

\section{Inquietudes y planteamientos recientes relativos a las cooperativas y el capital}

El Plan para una década cooperativa de la Alianza Cooperativa Internacional que ponía, al final del año 2012, año internacional de las cooperativas, a trabajar a todo el sector cooperativo en los años siguientes en unos temas, redactaba el quinto y último de ellos con estas palabras: "Conseguir capital fiable para las cooperativas al mismo tiempo que se garantice la gestión por parte de los miembros» ${ }^{18}$.

La Alianza está poniendo de manifiesto al proclamar este objetivo la necesidad de capital que tienen las cooperativas como cualquier otra empresa que debe financiar su actividad a corto y largo plazo, y que proviene en ellas de los socios que realizan sus aportaciones de forma directa o a través de los beneficios que quedan en forma de reservas, o de las instituciones crediticias convencionales.

El capital real, es decir, los medios de producción necesarios para la realización de una actividad económica, ha evolucionado exponen-

16 I.C.A.: Declaración..., o.c., p. 39.

17 Puede verse en I.C.A.: Declaración..., o.c., p. 43.

18 A.C.I.: Plan para una década cooperativa, enero de 2013, p. 6. 
cialmente desde el proceso de industrialización hasta la robotización actual, al tiempo que la dimensión de las empresas se ha multiplicado también en una economía globalizada. Esto hace que las inversiones iniciales de la cooperativa deban ser muy grandes y que la aportación de capital correspondiente para la financiación de esas inversiones por parte del socio haya de ser muy elevada. Y una cooperativa de una mayor dimensión, que se moviliza en mercados globalizados, necesita una mayor capacidad financiera para sus actividades presentes y futuras.

Si la cooperativa debe ser competitiva con el resto de las empresas ha de buscar la forma de obtener el capital necesario a lo largo del tiempo, y conseguir la permanencia de ese capital.

Pero, al mismo tiempo, en aplicación del principio, el capital tiene que ser gestionado democráticamente por los socios. Por eso mismo Johnston Birchall consideraba como una de las dos amenazas a los principios cooperativos, en la revisión que realizó diez años después de la vigencia de los mismos, la pérdida del principio de propiedad de los asociados en aquellas cooperativas en las que se obtiene capital de inversores externos a la cooperativa a cambio de acciones con derecho a voto ${ }^{19}$.

Para avanzar en el estudio del capital, la Alianza Cooperativa Internacional puso en marcha la Comisión de Expertos sobre Capital Cooperativo a finales de 2012, y ésta encargó al Filene Research Institute una investigación sobre el capital cooperativo, que se llevó a cabo bajo la dirección de A. Michael Andrews, dando lugar al Informe Investigación sobre el capital cooperativo 20 .

El Informe estudia los instrumentos y estructuras de capital que las cooperativas utilizan, las particularidades de las cooperativas pequeñas y nacientes, las estructuras de capital de las trescientas mayores cooperativas y mutuales, y las especialidades de las cooperativas financieras, para concluir que «acceder a capital adicional de los asociados o a capital de fuentes externas y mantener la adhesión a los principios cooperativos no son alternativas excluyentes» ${ }^{21}$.

Ahora bien, al mismo tiempo la Comisión de Expertos sobre Capital Cooperativo, considerando que el capital cooperativo se diferencia de otros tipos de capital en la medida en que las cooperativas están definidas y se identifican por un sistema de valores, principios y posiciones

19 BIRCHALL, Johnston: "Co-operative principles ten years on», Review of International Co-operation, I.C.A., Geneva, Vol. 98, N.² 2 / 2005, p. 61.

20 Publicado por la Alianza Cooperativa Internacional en 2015.

21 Andrews, A. Michael: Investigación sobre Capital Cooperativo. Alianza Cooperativa Internacional, julio de 2015, p. 43. 
filosóficas, y que el mundo busca modelos económicos equilibrados y sostenibles, contexto en el que el movimiento cooperativo tiene mucho que aportar, puso en marcha una nueva línea de trabajo para ir más lejos de la consideración técnica instrumental del capital y facilitar experiencias y opiniones que permitan avanzar en las reflexiones y debates posteriores. Este trabajo quedó plasmado en el documento titulado El dilema del capital en las cooperativas 22 .

Se trata de un conjunto amplio de aportaciones, desde las consideraciones filosóficas hasta las experiencias prácticas, para intentar situar la problemática del capital en las cooperativas y las vías de conducta que pueden orientar las soluciones en el momento presente.

En el Prólogo ${ }^{23}$ se recoge el planteamiento de que frente a la filosofía capitalista que se orienta al individuo y al mercado, la filosofía cooperativa se orienta a lo social y comunitario. De ello se desprende la importancia del trabajo frente al capital que queda supeditado al servicio del trabajo, mientras en la empresa capitalista es el capital el que contrata al trabajo, que queda supeditado a él.

La cooperativa se enfrenta a una doble problemática respecto al capital: por un lado está la naturaleza reembolsable del mismo que puede poner a la empresa en situaciones de falta de capital por su propia variabilidad; por otro, la falta de atractivo económico para el capital externo si no se maximiza el valor del accionista ni se realizan los retornos en función del capital aportado.

Ante esta problemática se orientan tres vías diferentes posibles, que pueden ser seguidas: la adaptación al mercado, el cambio del paradigma dominante a través de la identidad cooperativa, y el cambio del paradigma propio a través de la adaptación de los principios cooperativos.

La adaptación al mercado conservando los principios actuales se ha producido en la última década en la que se presentan cada vez más casos de cooperativas que se financian a través de nuevos instrumentos de capital. Por un lado se contempla la emisión de partes sociales no reembolsables y sin derecho a voto para ser suscritos por los asociados, pudiendo dichos partes ser negociables o no en el mercado interno de los propios socios. Por otro, en cooperativas de gran dimensión, el acceso de inversores externos a la cooperativa, manteniendo ésta el control democrático del capital.

22 Publicado por la Alianza Cooperativa Internacional en 2016.

23 Tan Suee Chieh y Chuin Ting Weber, "Prólogo del editor», en I.C.A.: El dilema del capital en las cooperativas, International Co-operative Alliance, 2016, pp. 12-20. 
La segunda vía se refiere al impulso para el cambio del paradigma capitalista dominante. La prolongada crisis económica y financiera ha puesto también en crisis al capitalismo moderno. En este contexto de búsqueda de alternativas al capitalismo las cooperativas tienen mucho que ofrecer. Desde la perspectiva de que las cooperativas ayudan a construir un mundo mejor el Plan para una década cooperativa pretende convertir a las cooperativas en el modelo empresa preferido en cuanto que sitúan a las personas en el centro de la empresa, unen a las comunidades y contribuyen a una mejor sostenibilidad. De este modo el movimiento cooperativo se pone en línea con las teorías del «nuevo capitalismo» que miran a los skateholders y a la comunidad.

Por fin, la tercera vía supone el cambio del propio paradigma cooperativo actual a través de la reflexión crítica sobre el modelo y una nueva evaluación de los principios cooperativos.

En esta perspectiva se propone dotar de una mayor vitalidad al sexto principio, de colaboración entre cooperativas, para facilitar la participación en el capital de unas cooperativas por parte de otras, la creación de fondos de estabilización en el movimiento cooperativo, y la asociación de cooperativas con mutuales y otras empresas de economía social.

$Y$, del mismo modo, se propone ampliar el séptimo principio, de interés por la comunidad, que profundiza en el desarrollo sostenible, para agrandar el filtro de la primacía del interés de los socios y llegar a otros interesados, aunque no sean los asociados, y a la sociedad en su conjunto: "Para ser auténticos defensores de la sostenibilidad tendríamos que extender nuestro cuidado por las personas a la custodia del planeta en el que viven y trabajan tanto esta generación como las generaciones futuras. En consecuencia, el capital y el beneficio no deben ser vistos como nuestros adversarios, sino como socios y facilitadores que contribuyen a la disciplina en la gestión y a promover la sostenibilidad financiera» 24 .

\section{Bibliografía}

A.C.I.: Plan para una década cooperativa, enero de 2013. En www.aciamericas.coop/lMG/pdf/ica_blueprint_es.pdf

A.C.I.: Notas de orientación para los principios cooperativos. Alianza Cooperativa Internacional, 2015. En www.aciamericas.coop/IMG/pdf/guidance_notes_es.pdf

24 Tan Suee Chieh y Chuin Ting Weber, «Prólogo del editor», en I.C.A.: El dilema..., o.c., p. 20. 
Andrews, A. Michael: Investigación sobre Capital Cooperativo. Alianza Cooperativa Internacional, julio de 2015. En www.aciamericas.coop/IMG/pdf/investigacion_sobre_capital.pdf

Birchall, Johnston: "Co-operative principles ten years on», Review of International Co-operation, I.C.A., Geneva, Vol. 98, N. ${ }^{\circ} 2$ / 2005, pp. 45-63.

Böök, Sven Ake: Co-operative values in a changing world (report to the ICA Congress, Tokio, October 1992), International Co-operative Alliance, Geneva, 1992.

I.C.A.: Declaración de la Alianza Cooperativa Internacional sobre la Identidad Cooperativa. Los principios cooperativos, Consejo Superior de Cooperativas de Euskadi, Vitoria-Gasteiz, 1996.

I.C.A.: El dilema del capital en las cooperativas, International Co-operative Alliance, 2016. En https://ica.coop/sites/default/files/publication-files/ica-eldilema-del-capital-en-las-cooperativas-1135397776.pdf

LAMBERT, Paul: La doctrina cooperativa, 3. ${ }^{a}$ ed., Intercoop, Buenos Aires, 1970.

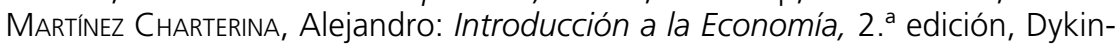
son, Madrid, 2017.

Martínez CharTerina, Alejandro: La cooperativa y su identidad, Dykinson, Madrid, 2016.

Laws and objets of the Rochdale Society of Equitable Pionners: enrolled according to the acts, $10^{\text {th }}$, George $I V$, and $4^{\text {th }}$ and $5^{\text {th }}$, William IV. Printed by Jesse Hall, Rochdale, 1844.

Supplement of Amended Laws of the First Department of the Rochdale Society of Equitable Pioneers, held in Toad-Lane, in the Parish of Rochdale, in the County of Lancaste. Printed by Jesse Hall, Rochdale, 1845. 